\title{
A modeling study on the signal transformation for the microsaccade generation
}

\author{
Keiichiro Inagaki ${ }^{*}$, Yutaka Hirata ${ }^{3}$, Shiro Usui ${ }^{1,2}$ \\ From Nineteenth Annual Computational Neuroscience Meeting: CNS*2010 \\ San Antonio, TX, USA. 24-30 July 2010
}

During visual fixation, stationary images are thought to be input to the visual system. However the actual input images are continuously fluctuating due to miniature eye movements. The miniature eye movements consist of microsaccade, drift, and tremor [1]. To date, while the functional roles of the miniature eye movements in perception, and their kinematic properties are gradually understood [1-3]; the mechanisms of their generation remain unknown. Here we focused on microsaccade, and constructed a model to explore the mechanisms of microsaccade generation.

\section{Methods}

Several lines of evidence assure that microsaccades share the same neuronal circuitry with saccades as they follow continuum behavioral profiles called the main sequence [1]. In the saccade related pathway, saccade commands generated in superior colliculus are relayed to motoneurons via burst neurons $(\mathrm{BN})$ that are tonically inhibited by omnipause neurons (OPN) except when saccade (i.e. fixation). Thus, we constructed a model based on the saccade model by Seung et al. [5] as it described explicitly excitatory/inhibitory BNs, the integrator network, and the oculomotor plant. We added OPN as a gain element, and controlled BNs activities to replicate the inter-microsaccade interval [4] and direction of microsaccade [6].

In simulation, our model successfully reproduced both horizontal and vertical microsaccades which were characterized by "square-jerk" or "single sided". Our model
A

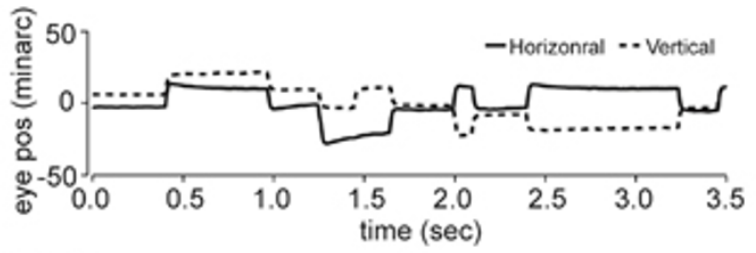

$\mathrm{B}$

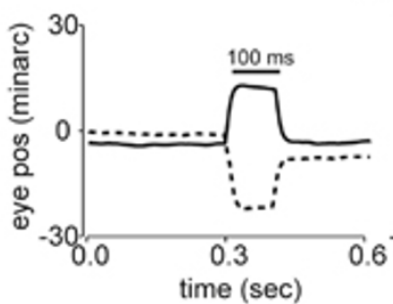

30 ,

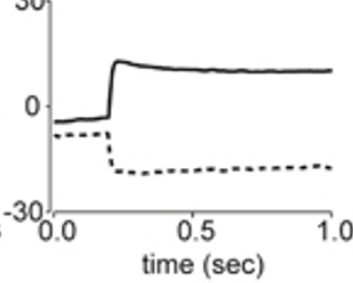

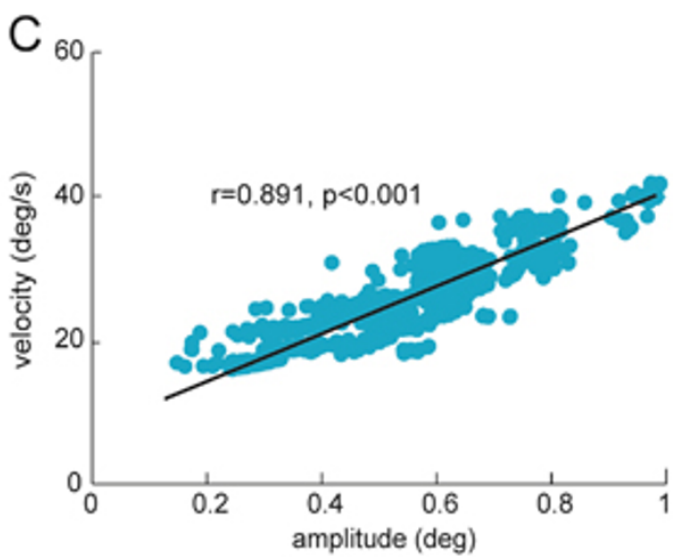

Figure 1 Simulation results. A: Fixating eye movement, B: Square-jerk wave (left) and Single sided (right) microsaccade, C: Main sequence. Solid and dashed line in A and B indicate horizontal or vertical eye movement. In C, solid line is linear regression line; $r$ and $p$ are multiple correlation coefficients and its significance.

* Correspondence: kay@brain.riken.jp

${ }^{1}$ Computational science research program, RIKEN, Wako, Saitama, 351-0198,

Japan 
also reproduced the main sequence characterized by the linear relationship between microsaccade amplitudes and peak velocities that is observed in the behavioral experiment (e.g. [8]). Our result suggested that the tonic inhibition from OPN plays a key role in the generation of microsaccades in depressing the activity of BNs that is the source of saccade/microsaccade deriving from superior colliculus. Nakao et al. reported that the BNs fired spikes at a low rate during fixation where the inhibition from the OPN exists [7], and those activities were replicated in our model as decreasing of firing rate of BNs. We conclude that those low rate spikes of BNs might be key activities to drive microsaccades. Figure 1

\section{Acknowledgements}

This research was supported by "The Next Generation Integrated Simulation of Living Matter" part of the Development and Use of the Next-Generation Supercomputer Project of the Ministry of Education, Culture, Sports and Technology.

\section{Author details}

${ }^{1}$ Computational science research program, RIKEN, Wako, Saitama, 351-0198, Japan. ${ }^{2}$ Laboratory for Neuroinformatics, RIKEN Brain science institute, Wako, Saitama, 351-0198, Japan. ${ }^{3}$ Department of computer science, Chubu University, Kasugai, Aichi, 487-8501, Japan.

Published: 20 July 2010

\section{References}

1. Martinez-Conde S, Macknik SL, Hubel DH: The role of fixational eye movements in visual perception. Nat Rev Neruosci 2004, 5:229-240.

2. Engbert $R$, Kliegl R: Microsaccades keep the eyes' balance during fixation. Psychol Sci 2004, 15:431-436.

3. Rucci M, lovin R, Poletti M, Santini F: Miniature eye movements enhance fine spatial detail. Nature 2007, 447:851-854

4. Otero-Millan J, Troncoso XG, Macknik SL, Serrano-Pedraza I, MartinezConde S: Saccades and microsaccades during visual fixation, exploration, and search: foundations for a common saccadic generator. J Vis 2009, 8(14):21.

5. Seung HS, Lee DD, Reis BY, Tank DW: Stability of the memory of eye position in a recurrent network of conductance-based model neurons. Neuron 2000, 26:259-271

6. Krauskopf J, Cornsweet TN, Riggs LA: Analysis of eye movements during monocular and binocular fixation. J. Opt. Soc. Am 1960, 50:572-578.

7. Nakao S, Shiraishi Y, Oda H, Inagaki M: Direct inhibitory projection of pontine omnipause neuron to burst neurons in the Forel's field $\mathrm{H}$ controlling vertical eye movement-related motoneurons in the cat. Exp Brain Res 1988, 70:632-636.

doi:10.1186/1471-2202-11-S1-P115

Cite this article as: Inagaki et al.: A modeling study on the signal transformation for the microsaccade generation. BMC Neuroscience 2010 11(Suppl 1):P115.

\section{Submit your next manuscript to BioMed Central and take full advantage of:}

- Convenient online submission

- Thorough peer review

- No space constraints or color figure charges

- Immediate publication on acceptance

- Inclusion in PubMed, CAS, Scopus and Google Scholar

- Research which is freely available for redistribution

Submit your manuscript at www.biomedcentral.com/submit 